Real Analysis Exchange

Vol. 22(1), 1996-97, pp. 406-412

Andrzej Nowik, Warsaw University, ul. Banacha 2, 02-097 Warsaw, Poland, e-mail: matan@@paula.univ.gda.pl

\title{
REMARKS ABOUT A TRANSITIVE VERSION OF PERFECTLY MEAGER SETS
}

\begin{abstract}
We show that if $X$ has the property that every continuous image into Baire space is bounded and $2^{\omega}$ is not a continuous image of $X$, then $X$ is always of first category in some additive sense. This gives an answer to an oral question of L. Bukovský, whether every wQN set has the latter property.
\end{abstract}

\section{Notation and Definitions}

$\mathcal{M G \mathcal { R }}(P)$ denotes the family of first category subsets of $P$. If $s \in 2^{<\omega}$ then $\mathcal{N}_{s}=\left\{t \in 2^{\omega}: t \supseteq s\right\}$ is a basic clopen set in $2^{\omega}$. Every clopen subset of $2^{\omega}$ is a finite union of $\mathcal{N}_{s}$. We denote by $\Delta_{1}^{0}\left(2^{\omega}\right)$ the class of clopen subsets of $2^{\omega}$.

Definition 1 [NSW] $X \subseteq 2^{\omega}$ is perfectly meager in the transitive sense iff for every perfect $P \subseteq 2^{\omega}$ one can find $\overline{F_{n}}=F_{n} \subseteq 2^{\omega}$ such that

$$
X \subseteq \bigcup_{n<\omega} F_{n}
$$

and

$$
\forall_{h \in 2^{\omega}}\left(\bigcup_{n<\omega} F_{n}\right) \cap(P+h) \in \mathcal{M} \mathcal{G} \mathcal{R}(P+h) .
$$

We use the abbreviation AFC' for this property.

Key Words: Hurewicz property, $S_{1}(\Gamma, \Gamma)$, AFC' set, wQN set, $\Sigma$ set, $\mathcal{H}$ property

Mathematical Reviews subject classification: Primary: 03E05 04A20 54D20

Received by the editors March 6, 1996

* This paper was supported by the KBN grant 2 P03A 04709 
Definition 2 [BRR] $X \subseteq 2^{\omega}$ is $w Q N$ iff for every sequence

$$
f_{n}: X \rightarrow \mathbf{R}_{+}
$$

of continuous functions converging to 0 we can find subsequence

$$
f_{n_{k}}: X \rightarrow \mathbf{R}_{+}
$$

such that one can find partition

$$
\left\{X_{n}\right\}_{n<\omega}
$$

of $X$ such that for every $n<\omega\left\{f_{n_{k}}\right\}_{k<\omega}$ converges uniformly to 0 on $X_{n}$.

Definition 3 [BRR] $X \subseteq 2^{\omega}$ is $\Sigma$ set iff for every sequence

$$
f_{n}: X \rightarrow \mathbf{R}_{+}
$$

of continuous functions converging to zero one can find subsequence

$$
f_{n_{k}}: X \rightarrow \mathbf{R}_{+}
$$

such that

$$
\forall x \in X \sum_{k<\omega} f_{n_{k}}(x)<\infty .
$$

We use also abbreviation $(C B)$ to denote the property of $X$ such that:

1. The image of the set $X$ by every continuous function into $\omega^{\omega}$ is bounded, and

2. The space $2^{\omega}$ is not continuous image of $X$.

The property of $X$ that every Borel image of $X$ into $\omega^{\omega}$ is bounded was considered in $[\mathrm{BJ}]$ and was there denoted by the property $\mathcal{H}$.

The property of $X$ that every continuous image of $X$ into $\omega^{\omega}$ is bounded is known as a Hurewicz property.

\section{Remarks}

In $[\mathrm{NSW}]$ authors proved, answering a question in $[\mathrm{S}]$, that every algebraic sum of sets of strong measure zero and strong first category has the Marczewski property $s_{0}$. In fact, authors proved, that every algebraic sum of so called AFC' set and strong measure zero set has the property $s_{0}$. In [NSW] it is proved that every $\gamma$ set has the property AFC'. It is obvious that every AFC' set is also perfectly meager. We know, that every $w Q N$ set is perfectly meager and every $\gamma$ set is $w Q N$. So it is natural question about relation between the class of $w Q N$ sets and AFC' sets. 


\section{Main Theorem}

Lemma 1 For every finite sequence of perfect subsets of $2^{\omega}: P_{1}, \ldots, P_{k} \subseteq 2^{\omega}$ there exists a partition of $2^{\omega}$ into two disjoint clopen sets: $U_{0}, U_{1}$ such that

$$
\forall_{h \in 2^{\omega}} \forall_{1 \leq i \leq k} \forall_{0 \leq j \leq 1}\left(h+P_{i}\right) \cap U_{j} \neq \emptyset .
$$

Proof. Moving each one of the sets $P_{i}(1 \leq i \leq k)$ we can assume, that each of them contains a null sequence: $\underline{0}=(0,0, \ldots)$.

Now there exists $n<\omega$ and $f_{i} \in P_{i} \backslash\{\underline{0}\}$ such, that $\left\{f_{i} \mid n\right\}_{i=1, \ldots, k}$ is a sequence of linear independent vectors over the field $Z_{2}=\{0,1\}$. (We treat $2^{n}$ as a linear space over the field $Z_{2}$ ). So we complete $\left\{f_{i} \mid n\right\}_{i=1, \ldots, k}$ to a base of the space $2^{n}$ with vectors $e_{k+1}, \ldots, e_{n} \in 2^{n}$.

Moreover, we put $e_{i}=f_{i} \mid n(i=1, \ldots, k)$. So $\left(e_{1}, \ldots, e_{n}\right)$ is a base of $2^{n}$ over $Z_{2}$.

Now we consider

$$
V:=\left\{\sum_{i=1}^{n} \alpha_{i} e_{i}: \alpha_{i} \in Z_{2} \wedge\left|\left\{i: \alpha_{i}=1\right\}\right| \text { is even }\right\} .
$$

Obviously $V$ is a linear subspace of $2^{n}$ over a field $Z_{2}$, moreover is has a codimension one. Also it is clear, that $V$ does not contain $e_{i}=f_{i} \mid n(1 \leq i \leq k)$.

Now we see, that for every $s \in 2^{n}$

$$
s \in V \quad \text { iff } \quad s+f_{i} \mid n \notin V .
$$

It is easy to see now, that if we define:

$$
U_{0}:=\bigcup_{s \in V} \mathcal{N}_{s} \text { and } U_{1}:=\bigcup_{s \in 2^{n} \backslash V} \mathcal{N}_{s}
$$

then $U_{0}$ and $U_{1}$ will be disjoint clopen sets having the properties of Lemma 1.

Lemma $2^{\prime}$ Let $P$ be a perfect set and let $\left\{B_{i}\right\}_{i<\omega}$ be an enumeration of the base of $P$ with $B_{0}=P$. There is a system $\left\{U_{s}: s \in 2^{<\omega}\right\}$ of clopen subsets of $2^{\omega}, U_{\emptyset}=2^{\omega},\left\{U_{s \frown\langle 0\rangle}, U_{s \frown\langle 0\rangle}\right\}$ is a partition of $U_{s}$ such that

$$
\text { (1) } \forall_{s \in 2<\omega} \forall_{h \in 2^{\omega}} \forall_{j=0,1} \forall_{i \leq|s|}\left(B_{i}+h\right) \cap U_{s} \neq \emptyset \rightarrow\left(B_{i}+h\right) \cap U_{s}<\langle j\rangle \neq \emptyset \text {. }
$$

Proof. By induction on length of $s \in 2^{<\omega}$ we define the sets $U_{s}$. We set $U_{\emptyset}=2^{\omega}$ and assuming that $U_{s}$ are constructed for all $s \in 2^{k}$ we find an integer 
$n_{k}$ such that for every $s \in 2^{k}$ there is a set $S_{s} \subseteq 2^{n_{k}}$ such that $U_{s}=\bigcup_{t \in S_{s}} \mathcal{N}_{t}$. For $s \in 2^{k}$ we set

$$
\begin{aligned}
T_{k} & =\left\{h \in 2^{\omega}: \forall_{n \geq n_{k}} h(n)=0\right\}, \\
R_{s} & =\left\{\left(B_{i}+h\right) \cap U_{s}:\left(B_{i}+h\right) \cap U_{s} \neq \emptyset, i \leq k, h \in T_{k}\right\} .
\end{aligned}
$$

Let $\left\{U_{0}^{s}, U_{1}^{s}\right\}$ be a clopen partition of $2^{\omega}$ with properties ensured by Lemma 1 for the finite system of perfect sets $R_{s}$ and let us set

$$
U_{s \frown\langle j\rangle}=U_{s} \cap U_{j}^{s}, \quad j=0,1 .
$$

Now if $h \in 2^{\omega}$ is arbitrary, let $h_{(k)}, h^{(k)} \in 2^{\omega}$ be such that $h_{(k)}\left|n_{k}=h\right| n_{k}$, $h^{(k)}\left|\left[n_{k}, \infty\right)=h\right|\left[n_{k}, \infty\right)$, and $h_{(k)}+h^{(k)}=h$. Hence $h_{(k)} \in T_{k}$. If $\left(B_{i}+h\right) \cap$ $U_{s} \neq \emptyset$, then also $\left(B_{i}+h_{(k)}\right) \cap U_{s} \neq \emptyset$, because $U_{s}+h^{(k)}=U_{s}$. Therefore

$$
\left(B_{i}+h\right) \cap U_{s \frown\langle j\rangle}=\left[\left(\left(B_{i}+h_{(k)}\right) \cap U_{s}\right)+h^{(k)}\right] \cap U_{j}^{s} \neq \emptyset
$$

and condition (1) is fulfilled.

Lemma $2^{\prime}$ has the following equivalent reformulation.

Corollary $2^{\prime \prime} \quad$ For every perfect set $P \subseteq 2^{\omega}$ there is a continuous mapping $\Phi: 2^{\omega} \rightarrow 2^{\omega}$ such that for each $h \in 2^{\omega}$ the restriction $\Phi \mid(P+h)$ is an open mapping from $P+h$ onto $2^{\omega}$.

Proof. Let $\left\{U_{s}: s \in 2^{<\omega}\right\}$ be a system of clopen subsets of $2^{\omega}$ as is stated in Lemma $2^{\prime}$. Let us define $\Phi: 2^{\omega} \rightarrow 2^{\omega}$ by $\Phi(x)=y$ iff $x \in \bigcap_{n<\omega} U_{y \mid n}$. Taking $i=0$ in condition (1) we obtain

(2) $\forall_{h \in 2^{\omega}} \forall_{s \in 2}<\omega(P+h) \cap U_{s} \neq \emptyset$

which easily implies that the mapping $\Phi \mid(P+h)$ is onto $2^{\omega}$. Similarly, condition (1) implies that

$$
\Phi\left(B_{i}+h\right)=\bigcup\left\{\mathcal{N}_{s}: s \in 2^{i},\left(B_{i}+h\right) \cap U_{s} \neq \emptyset\right\} .
$$

Corollary $2^{\prime \prime \prime}$ If $X \subseteq 2^{\omega}$ and $2^{\omega}$ is not a continuous image of $X$, then for every perfect set $P \subseteq 2^{\omega}$ there is a sequence $\left\{U_{i}\right\}_{i<\omega}$ of disjoint clopen subsets of $2^{\omega}$ such that

(1) $\forall_{h \in 2^{\omega}} \forall_{j<\omega}(P+h) \cap U_{j} \neq \emptyset$,

(2) $\forall_{h \in 2^{\omega}}(P+h) \backslash \bigcup_{j<\omega} U_{j} \in \mathcal{M G \mathcal { R }}(P+h)$, 
(3) $X \subseteq \bigcup_{i<\omega} U_{i}$.

Proof. Let $\Phi: 2^{\omega} \rightarrow 2^{\omega}$ be a continuous mapping such that $\Phi \mid(P+h)$ is open and onto $2^{\omega}$ for every $h \in 2^{\omega}$. Take any $y \in 2^{\omega} \backslash \Phi(X)$. In particular, $\Phi^{-1}\left(2^{\omega} \backslash\{y\}\right) \cap(P+h)$ is open dense in $P+h$. Let $S \subseteq\left\{s \in 2^{<\omega}: y \notin \mathcal{N}_{s}\right\}$ be a maximal antichain in $2^{<\omega}$ and let $\left\{U_{i}\right\}_{i<\omega}$ be an enumeration of the set $\left\{\Phi^{-1}\left(\mathcal{N}_{s}\right): s \in S\right\}$.

Theorem 1 Let $X \subseteq 2^{\omega}$ be a set with the property $(C B)$. Then $X$ has the property $A F C$ '.

Proof. Let $P \subseteq 2^{\omega}$ be a perfect set. Let

$$
\left\{B_{i}\right\}_{i<\omega}
$$

be a clopen base in $P$. For every $j<\omega$ we apply Corollary $2^{\prime \prime \prime}$ to the perfect set $B_{j}$ and we obtain a sequence

$$
\left\{U_{i}^{(j)}\right\}_{i<\omega}
$$

of clopen subsets of $2^{\omega}$. Put

$$
N=\bigcap_{j<\omega} \bigcup_{i<\omega} U_{i}^{(j)} .
$$

We define now:

$$
\Psi: N \rightarrow \omega^{\omega}
$$

by the condition, that

$$
\Psi(x)=f \Longleftrightarrow x \in \bigcap_{j<\omega} U_{f(j)}^{(j)}
$$

for $x \in N$ and $f \in \omega^{\omega}$.

Now $\Psi(X)$ is bounded in $\omega^{\omega}$, so there exists a sequence $f_{n} \in \omega^{\omega}, n<\omega$, such that the closed sets

$$
F_{n}=\bigcap_{j<\omega} \bigcup_{i<f_{n}(j)} U_{i}^{(j)} \subseteq N
$$

cover $X$.

Take any $h \in 2^{\omega}, n<\omega$ and assume that $F_{n} \cap(P+h)$ is not meager in $P+h$, i.e. there exists $j<\omega$ such that $B_{j}+h \subseteq F_{n}$. By condition (1) of Corollary $2^{\prime \prime \prime}$ we can now choose $i>f_{n}(j)$ so that $\left(B_{j}+h\right) \cap U_{i}^{(j)} \neq \emptyset$ and 
then $F_{n} \cap U_{i}^{(j)} \neq \emptyset$ which is a contradiction because $F_{n}$ is disjoint with every set $U_{i}^{(j)}$ for $i \geq f_{n}(j)$.

This gives us that

$$
\forall_{n<\omega} F_{n} \cap(P+h) \in \mathcal{M G \mathcal { R }}(P+h)
$$

holds true and so $X$ has the property AFC'.

\section{Conclusions}

Conclusion 1 Every $\Sigma$ set is an $A F C^{\prime}$ set.

PRoof. We know from [BRR], that every continuous image of an wQN set into $\omega^{\omega}$ is bounded. Modifying the proof one can obtain, that continuous image of a $\Sigma$ into $\omega^{\omega}$ is also bounded. From [BRR] we know that continuous image of a $\Sigma$ set is also a $\Sigma$ set. One can show, that $2^{\omega}$ is not a $\Sigma$ set, so we obtain, that every $\Sigma$ set has the property $(C B)$.

Conclusion 2 Every $w Q N$ subset of $2^{\omega}$ is an $A F C^{\prime}$ set.

Proof. By $[\mathrm{BRR}]$ every $w Q N$ set is a $\Sigma$ set.

Conclusion 3 Every $\mathcal{H}$ set is $A F C$ '.

Proof. Every $\mathcal{H}$ set is a wQN set.

\section{Conclusion 4}

$$
\operatorname{non}\left(A F C^{\prime}\right) \geq \mathbf{b} .
$$

Proof. Obvious, because every $X$ with the cardinality less than $\mathbf{b}$ has the property $(C B)$.

Conclusion 5 Every $S_{1}(\Gamma, \Gamma)$ set is $A F C^{\prime}$. Recall that $X$ is $S_{1}(\Gamma, \Gamma)$ iff for every sequence $\mathcal{U}_{n}$ of open $\gamma$ covers we can find $V_{n} \in \mathcal{U}_{n}$ such that $\left\{V_{n}\right\}_{n<\omega}$ is also a $\gamma$ cover. Under $\gamma$ cover we mean every cover $\mathcal{U}$ of $X$ such that $|\mathcal{U}| \geq \omega$ and

$$
\forall_{x \in X}|\{U \in \mathcal{U}: x \notin U\}|<\omega .
$$

This notion was defined and considered in [JMSS].

PROOF. In [JMSS] authors proved that every $S_{1}(\Gamma, \Gamma)$ set has the Hurewicz property and also that $2^{\omega}$ has not the property $S_{1}(\Gamma, \Gamma)$ ([JMSS, Theorem 2]). Because every continuous image of set of the property $S_{1}(\Gamma, \Gamma)$ has also the property $S_{1}(\Gamma, \Gamma)$ ([JMSS, Theorem 3.1]), so we obtain the Conclusion 5 . 
Conclusion 6 There exists in $Z F C$ an $A F C$ ' uncountable set.

Proof. In [JMSS, proof of Theorem 5.1] the existence of an uncountable set in $S_{1}(\Gamma, \Gamma)^{*}$ is proved and I. Recław $[\mathrm{R}]$ proved that every such set is a wQN set.

Acknowledgment: The author would like to express his gratitude to the referee for a very nice version of Lemma 2'.

\section{References}

[BJ] T. Bartoszyński and H. Judah, Borel images of sets of reals, Real Analysis Exchange, 20 (1994-95), 536-558.

[BRR] L. Bukovský, I. Recław and M. Repický, Spaces not distinguishing pointwise and quasinormal convergence of real functions, Topology and its Applications, 41 (1991), 25-40.

[JMSS] W. Just, A. Miller, M. Scheepers and P. J. Szeptycki, The combinatorics of open covers (II), Topology and its Applications (to appear).

[NSW] A. Nowik, M. Scheepers and T. Weiss, The algebraic sum of sets of real numbers with strong measure zero sets, to appear in Journal of Symbolic Logic.

[R] I. Recław, A note on QN-sets and wQN-sets, preprint, 1996.

[S] M. Scheepers, Additive properties of sets of real numbers and an infinite game, Quaestiones Mathematicae, 16 (1993), 177-191. 